The Chittagong Univ. J. Sci. 43(1): 93-112, 2021

\title{
Factors Influencing the Awareness of HIV/AIDS among Ever Married Women in Bangladesh
}

\author{
Md. Abdul Karim ${ }^{1 *}$, Mohammad Omar Faruk ${ }^{2}$, Mohammad Shohel Rana² \\ ${ }^{1}$ Department of Statistics, University of Chittagong, Chittagong-4331, Bangladesh \\ ${ }^{2}$ Department of Statistics, Noakhali Science and Technology University, Noakhali -3814, Bangladesh \\ "Corresponding author: makstatcu@yahoo.com
}

Manuscript Submitted on 15/02/2021, Revised manuscript received on 05/10/2021, and accepted on 10/10/2021.

\begin{abstract}
The main focus of this research study is to investigate the determining factors affecting the awareness of HIV/AIDS among ever married women in Bangladesh. To accomplish this task fruitfully, the study uses the nationally representative BDHS 2014 data set. The finding of this study illustrates that about $70 \%$ of the respondents have heard of AIDS. Among the respondents who have heard of AIDS, $97.4 \%$ and $92.5 \%$ of them have HIV/AIDS transmission and prevention knowledge respectively. Bivariate analysis states that the variables type of place of residence, division, respondents' education and access to mass media are significantly associated with the awareness of HIV/AIDS and also its transmission and prevention knowledge. The multivariate hierarchical logistic regression analysis reveals that respondents' education has a significant impact on HIV/AIDS awareness and its transmission and prevention knowledge. The odds ratio in all the models indicates that respondents who are highly educated and have access to mass media are more knowledgeable about HIV/AIDS. Type of place of residence and region has significant effect on the awareness of HIV/AIDS, and also its transmission and prevention knowledge. Age of the respondents, husband education and occupation also have significant impact on the awareness of HIV/AIDS. Finding of this study further reflects that use of contraceptives is a predominant significant factor influencing the awareness of HIV/AIDS. Therefore, based on the findings it can be concluded that appropriate steps should be launched across the country to raise the awareness level of such a deadly disease for the reduction of its spread in Bangladesh.
\end{abstract}

Keywords: HIV/AID, Awareness, Transmission, Prevention, Virus, $\chi^{2}$ test statistic, Hierarchical logistic regression.

DOI: https://doi.org/10.3329/cujs.v43i1.57338 
এই গবেষণা সমীক্ষারমূল কেন্দ্রবিন্দু হলো বাংলাদেশের বিবাহিত মহিলাদের মধ্যে HIV/AIDS সচেতনাকে প্রভাবিতকারী গুণনিয়ক গুলি অনুসন্ধান করা। এই কাজটি ফলপ্রসূভাবে সম্পাদন করতে অধ্যয়নটিতে জাতীয় প্রতিনিধিত্বকারী BDHS-২০১৪ ডেটা সেট ব্যবহার করা হয়। এই সমীক্ষার অনুসন্ধানে প্রমানিত হয়েছে যে, প্রায় ৭০\% উত্তরদাতা এইডস সম্মন্ধে সচেতন। যারা এইডস সমন্ধে শুনেছেন তাদের মধ্যে যথাক্রমে ৯৭.৪\% এবং ৯২.৫\% HIV/AIDS সংক্রমন এবং প্রতিরোধ সম্পর্কে জ্ঞান রাখে। দ্বি-চলক বিশ্লেষনে প্রতীয়মান হয় যে, আবাসমৃল, বিভাগ, উত্তরদাতাদের শিক্ষা এবং গণমাধ্যম ব্যবহারকারীগণ HIV/AIDS সম্পর্কে তাৎপর্যপূর্ণভাবে সচেতন এবং এর সংক্রমন ও প্রতিরোধ জ্ঞানের সাথে উল্লেখযোগ্যভাবে জড়িত। বহুচলক হায়ারারকিকাল লজিষ্টিক রিগ্রেশন বিশ্লেষনে প্রতীয়নমান হয়েছে যে, উত্তরদাতাদের শিক্ষা HIV/AIDS সচেতনতা এবং এর সংক্রমণ ও প্রতিরোধ জ্ঞানের উপর উল্লেখযোগ্য প্রভাব ফেলে। সমস্ত মডেলের অডস্ অনুপাত নির্দেশ করে যে, উচ্চ শিক্ষা এবং গণমাধ্যমে অ্যাক্সেস রয়েছে এমন উত্তরদাতারা HIV/AIDS সম্পর্কে আরো জ্ঞানবান। আবাসমৃল এবং বিভাগ HIV/AIDS সচেতনতা এবং এর সংক্রমন ও প্রতিরোধ জ্ঞানের উপর উল্লেখযোগ্য প্রভাব রয়েছে। উত্তরদাতাদের বয়স, স্বামীর শিক্ষা এবং পেশা HIV/AIDS সচেতনতার উপরও তাৎপর্যপূর্ণ প্রভাব রয়েছে। এই গবেষণার ফলাফনে আরো প্রতীয়মান হয় যে, গর্ভনিরোধক ব্যবহার HIV/AIDS সচেতনতার উপর একটি উল্লেখযোগ্য প্রভাব বিষ্তারকারী নির্ণায়ক। সুতরাং, ফলাফল এর উপর ভিত্তি করে উপসংহারে বলা যায় যে, বাংলাদেশে এর বিস্তার হ্রাসের জন্য এ জাতীয় মারাহ্মক রোগের সচেতনতা স্তর বাড়াতে দেশ জুড়ে যথাযথ পদক্ষেপ গ্রহণ করা জরুরী।

\section{Introduction}

Acquired Immune Deficiency Syndrome (AIDS) is a deadly disease lead to the death of human life. AIDS is an illness caused by a virus called HIV, which stands for Human Immunodeficiency Virus. Once someone infected with HIV the virus will remain into the body for the whole life. No cure or even no vaccine invented yet to prevent people from its infection. HIV attacks the CD4 cell (T cell), which help the immune system fight off the infection. In addition, HIV reduces the number of CD4 cells in the body, and gradually the human immune system become weak and unable to fight off other opportunistic disease. These opportunistic infections take the advantage of a very weak immune system and gradually lead to the disease like AIDS. Moreover, AIDS is the stage of infection that occurs when immune system is badly damaged and the human body becomes vulnerable to opportunistic infections and ultimately life gradually fall 
into tremendous risk of death. The first case of AIDS was officially avowed in the USA on June 5, 1981, since then the world experiencing a terrific health risk. At the end of 2015, 36.7 million people living with HIV worldwide [1]. Globally, the number of new HIV infection were 2.1 million and the number of death due to AIDS was 1.1 million in 2015. Since the beginning of this epidemic, the numbers of people becomes infected with AIDS is 78 million and a total of 35 million people have died due to AIDS in the world [2]. In Asia pacific, the estimated number of people living with HIV was 5.1 million and the ever prevalence rate is $0.2 \%$ in 2015 [3]. In 2015, the number of death due to AIDS was 0.18 million and the estimated number of new HIV infection was 0.3 million in Asia pacific [4]. As HIV/AIDS is a deadly epidemic in the current world and causing death in a great amount, so as this continuation, Bangladesh is not out of this consequence. Although, still Bangladesh is low prevalence country compared to other neighboring countries as well as other countries in the world, but attention should be kept on the spread of this dangerous epidemic and regular monitoring is essential. Different studies reported that the current prevalence rate of HIV/AIDS in Bangladesh is still remains less than 0.01\% [5-6]. The first case of HIV/AIDS in Bangladesh was detected in 1989 [7]. The total number of new HIV infection decreases from 445 in 2011 to 433 in 2014. However, the number of new AIDS case (51) remain same in both period but the number of death due to AIDS increases to 91 in 2014 from 84 in 2011 [89]. Thus, it is very important to know the current scenario of awareness, knowledge, and attitude towards the HIV/AIDS epidemic in Bangladesh. Keeping it in view, it is immensely important to extensively study the awareness of HIV/AIDS along with its transmission and prevention knowledge because this disease is vulnerable due to high risk factors in the densely populated country like Bangladesh. Therefore, this research paper attempts to extensively study the awareness of HIV/AIDS along with its transmission and prevention knowledge among ever married women in Bangladesh.

\section{Objectives of the Study}

The prime objective of this research study is to thoroughly investigate the awareness of HIV/AIDS, its transmission and prevention knowledge because it is one of the 
fatal diseases across the world. However, the specific objectives of this study are as follows:

* To observe the percentage distribution of the respondents by their background characteristics about the awareness of the HIV/AIDS, its transmission and prevention knowledge;

* To examine the association between the background characteristics of the respondents and the awareness of HIV/AIDS, its transmission and prevention knowledge;

* To identify the significant factors influencing the awareness of HIV/AIDS, its transmission and prevention knowledge among the ever married women in Bangladesh.

\section{Methodology}

This research work is based on the Bangladesh Demographic and Health Survey 2014 data set. The survey was carried out under the authority of national institute of population research and training (NIPORT). A detailed description of the data collection procedure and sample design for the survey can be obtained elsewhere [9]. In this survey, a total of 16,231 ever married women were interviewed out of 17,863 . First of all HIV/AIDS awareness level is measured from these 16,231 female respondents. Secondly, to assess the comprehensive transmission and prevention knowledge of HIV/AIDS, mutually exclusive sub-samples of 11,332 and 11,284 married women who have heard of HIV/AIDS are extracted respectively among all respondents. To examine the significant association between the HIV/AIDS awareness and available explanatory variables, $\chi^{2}$ statistic is computed after constructing contingency tables at $5 \%$ level of significance. Thereafter, the multivariate hierarchical logistic regression analysis was employed to identify the significant prognostic factors of HIV/AIDS awareness, transmission and prevention knowledge considering the variables found significant in bivariate analysis. In order to apply the hierarchical logistic regression in different models the variables considered in this analysis are categorized in three sub-groups accordingly and presented in the following framework. 


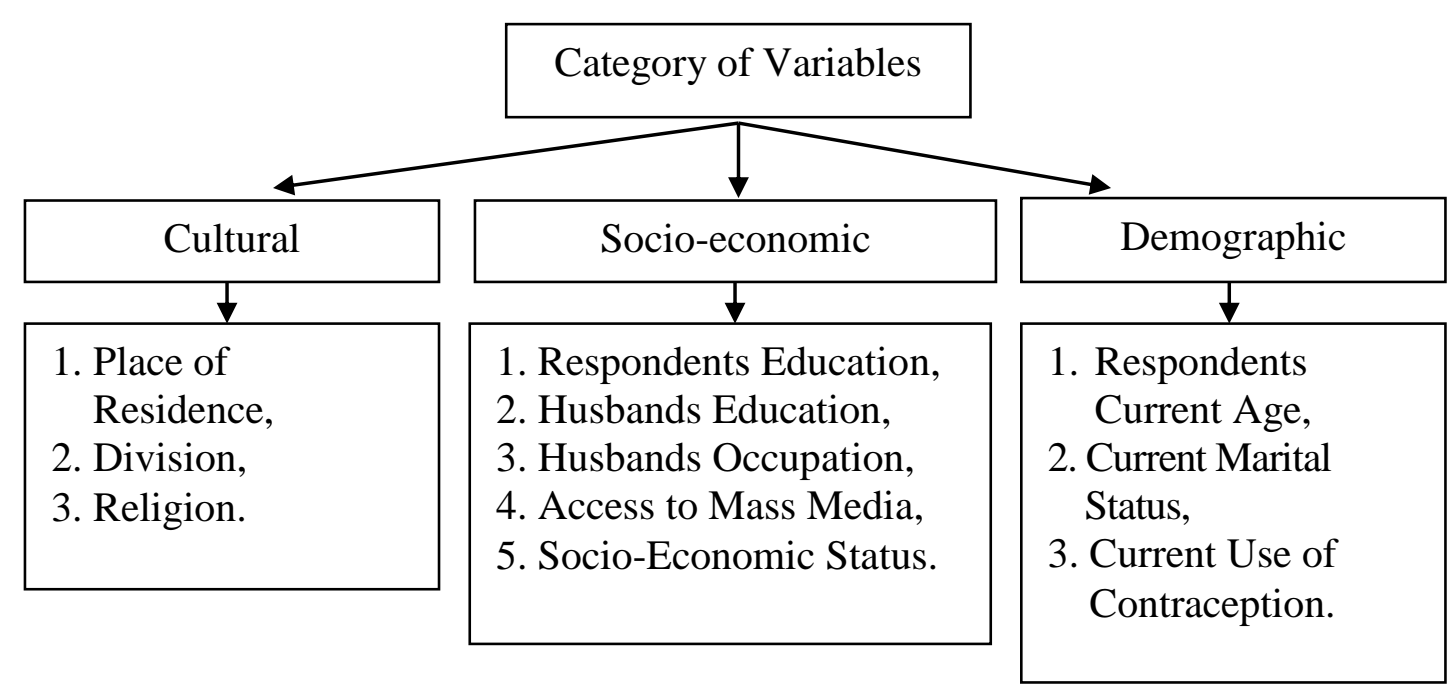

These groups of variables were entered into the regression in a stepwise fashion following a cultural, socioeconomic and demographic hierarchy of their effect on the dependent variables [10].

\section{Results and Discussion}

HIV/AIDS is one of the killer diseases in the world. So, in the context of Bangladesh it is very important to analyze thoroughly its awareness, transmission and prevention for the clear perception of the people. Therefore, at the very outset the percentage distribution of the sampled respondents regarding ever heard of HIV/AIDS by their background characteristics are analyzed and presented in the Table 1 . The result of the Table 1 shows that $70 \%$ of the respondents have heard of AIDS, which states that $70 \%$ of the respondents are aware of HIV/AIDS. Among the respondents who have heard of AIDS 97.4 \% of them are also know how HIV can be transmitted and 92.5\% know how to prevent it from transmission. The age of the respondents selected in this study are above 30 years $(54.4 \%)$ and $65.1 \%$ respondents live in rural areas. Findings in the Table 1 reveal that $17.3 \%$ of the respondents are from Dhaka division followed by Chittagong $(15.8 \%)$ and lowest in sylhet (11.8\%). In the context of education, only $9.2 \%$ respondents are highly educated whereas, $14.5 \%$ of their husbands are also highly educated. 
Table 1. Percentage distribution of the ever married respondents by background characteristics of ever heard of HIV/AIDS in Bangladesh.

\begin{tabular}{|c|c|c|c|}
\hline Background Characteristics & Categories & $\mathbf{N}$ & Percentage \\
\hline \multirow[t]{2}{*}{ Ever heard of AIDS } & No & 4876 & 30.0 \\
\hline & Yes & 11355 & 70.0 \\
\hline \multirow[t]{2}{*}{ HIV/AIDS transmission Knowledge } & No & 291 & 2.6 \\
\hline & Yes & 11041 & 97.4 \\
\hline \multirow[t]{2}{*}{ HIV/AIDS prevention knowledge } & No & 841 & 7.5 \\
\hline & Yes & 10443 & 92.5 \\
\hline \multirow[t]{4}{*}{ Respondents current Age } & $15-19$ & 1631 & 10.0 \\
\hline & $20-24$ & 2739 & 16.9 \\
\hline & $25-29$ & 3032 & 18.7 \\
\hline & $30+$ & 8829 & 54.4 \\
\hline \multirow[t]{2}{*}{ Type of place of residence } & Urban & 5667 & 34.9 \\
\hline & Rural & 10564 & 65.1 \\
\hline \multirow[t]{7}{*}{ Division } & Barisal & 1932 & 11.9 \\
\hline & Chittagong & 2571 & 15.8 \\
\hline & Dhaka & 2807 & 17.3 \\
\hline & Khulna & 2381 & 14.7 \\
\hline & Rajshahi & 2314 & 14.3 \\
\hline & Rangpur & 2307 & 14.2 \\
\hline & Sylhet & 1919 & 11.8 \\
\hline \multirow[t]{4}{*}{ Respondents education } & Illiterate & 3994 & 24.6 \\
\hline & Primary & 4813 & 29.7 \\
\hline & Secondary & 5935 & 36.6 \\
\hline & Higher & 1489 & 9.2 \\
\hline
\end{tabular}




\begin{tabular}{|c|c|c|c|}
\hline Background Characteristics & Categories & $\mathbf{N}$ & Percentage \\
\hline \multirow[t]{4}{*}{ Husbands education } & Illiterate & 4738 & 29.2 \\
\hline & Primary & 4474 & 27.6 \\
\hline & Secondary & 4669 & 28.8 \\
\hline & Higher & 2350 & 14.5 \\
\hline \multirow[t]{2}{*}{ Religion } & Islam & 14623 & 90.1 \\
\hline & Others & 1608 & 9.9 \\
\hline \multirow[t]{4}{*}{ Husbands occupation } & Agriculture & 4551 & 28.0 \\
\hline & Service & 4691 & 28.9 \\
\hline & Business & 3700 & 22.8 \\
\hline & Others & 3289 & 20.3 \\
\hline \multirow[t]{2}{*}{ Current marital status } & Married & 15258 & 94.0 \\
\hline & Separated & 973 & 6.0 \\
\hline \multirow[t]{2}{*}{ Current contraceptive method } & No & 6520 & 40.2 \\
\hline & Yes & 9711 & 59.8 \\
\hline \multirow[t]{3}{*}{ Socio-economic status } & Poor & 3701 & 22.8 \\
\hline & Middle & 8753 & 53.9 \\
\hline & Rich & 3777 & 23.3 \\
\hline \multirow[t]{2}{*}{ Access to mass media } & No & 6145 & 37.9 \\
\hline & Yes & 10086 & 62.1 \\
\hline Total & & 16231 & $100 \%$ \\
\hline
\end{tabular}

Result in Table 1 elucidates that $29.2 \%$ husbands of the respondents are illiterate. Percentage distribution also shows that $90.1 \%$ of the respondents are Muslim and maximum about $29 \%$ husbands of the respondents are service holder. The results of the study also show that $59.8 \%$ of the respondents use contraceptives. In Bangladesh, $53.9 \%$ of the respondents belong to middle socioeconomic stratum and only $62.1 \%$ have access to mass media. The findings at a glance reflect that the overall level of 
heard of HIV/AIDS is somewhat encouraging but the current level should be raised to protect them from this kind of fetal disease.

Besides percentage distribution of the respondents by background characteristics it is absolutely essential to examine whether there is any significant association between awareness, transmission and prevention knowledge of HIV/AIDS by explanatory variables considered in the analysis. Therefore, to investigate whether there is any association between awareness, transmission and prevention knowledge of HIV/AIDS by available explanatory variables, contingency tables and chi-square test statistic along with corresponding p-values are computed and given in the Table 2.

Table 2. Test of association between awareness of HIV/AIDS, its transmission and prevention knowledge by background characteristics of the respondents.

\begin{tabular}{|c|c|c|c|c|c|c|c|c|c|c|c|c|c|}
\hline \multirow{2}{*}{\multicolumn{2}{|c|}{$\begin{array}{c}\text { Background } \\
\text { characteristics }\end{array}$}} & \multicolumn{4}{|c|}{$\begin{array}{c}\text { Ever heard } \\
\text { of HIV/AIDS }\end{array}$} & \multicolumn{4}{|c|}{$\begin{array}{c}\text { HIV/AIDS transmission } \\
\text { knowledge }\end{array}$} & \multicolumn{4}{|c|}{$\begin{array}{c}\text { HIV/AIDS prevention } \\
\text { knowledge }\end{array}$} \\
\hline & & $\begin{array}{l}\text { No } \\
(\%)\end{array}$ & $\begin{array}{l}\text { Yes } \\
(\%)\end{array}$ & $\chi^{2}$ & P-value & $\begin{array}{l}\text { No } \\
(\%)\end{array}$ & $\begin{array}{l}\text { Yes } \\
(\%)\end{array}$ & $\chi^{2}$ & P-value & $\begin{array}{l}\text { No } \\
(\%)\end{array}$ & $\begin{array}{l}\text { Yes } \\
(\%)\end{array}$ & $\chi^{2}$ & P-value \\
\hline \multirow{4}{*}{$\begin{array}{l}\text { Respondents } \\
\text { current age }\end{array}$} & $15-19$ & 28.4 & 71.6 & \multirow{4}{*}{275.90} & \multirow{4}{*}{.000} & 2.4 & 97.6 & \multirow{4}{*}{5.55} & \multirow{4}{*}{.135} & 7.2 & 92.8 & \multirow{4}{*}{4.56} & \multirow{4}{*}{.207} \\
\hline & $20-24$ & 22.2 & 77.8 & & & 2.2 & 97.8 & & & 8.1 & 91.9 & & \\
\hline & $25-29$ & 22.7 & 77.3 & & & 2.2 & 97.8 & & & 6.5 & 93.5 & & \\
\hline & $30+$ & 35.3 & 64.7 & & & 2.9 & 97.1 & & & 7.6 & 92.4 & & \\
\hline \multirow{2}{*}{$\begin{array}{l}\text { Type of place } \\
\text { of residence }\end{array}$} & Urban & 17.0 & 83.0 & \multirow{2}{*}{699.60} & \multirow{2}{*}{.000} & 2.0 & 98.0 & \multirow{2}{*}{9.44} & \multirow{2}{*}{.001} & 6.5 & 93.5 & \multirow{2}{*}{9.98} & \multirow{2}{*}{.001} \\
\hline & Rural & 37.0 & 63.0 & & & 3.0 & 97.0 & & & 8.1 & 91.9 & & \\
\hline \multirow{7}{*}{ Division } & Barisal & 27.3 & 72.7 & \multirow{7}{*}{351.07} & \multirow{7}{*}{.000} & 2.4 & 97.6 & \multirow{7}{*}{53.81} & \multirow{7}{*}{.000} & 6.7 & 93.3 & \multirow{7}{*}{67.79} & \multirow{7}{*}{.000} \\
\hline & Chittagong & 29.7 & 70.3 & & & 2.4 & 97.6 & & & 9.4 & 90.6 & & \\
\hline & Dhaka & 22.8 & 77.2 & & & 2.4 & 97.6 & & & 4.6 & 95.4 & & \\
\hline & Khulna & 21.2 & 78.8 & & & 1.7 & 98.3 & & & 8.9 & 91.1 & & \\
\hline & Rajshahi & 34.0 & 66.0 & & & 1.9 & 98.1 & & & 5.1 & 94.9 & & \\
\hline & Rangpur & 39.4 & 60.6 & & & 2.6 & 97.4 & & & 8.7 & 91.3 & & \\
\hline & Sylhet & 38.9 & 61.1 & & & 5.6 & 94.4 & & & 9.9 & 90.1 & & \\
\hline \multirow{4}{*}{$\begin{array}{l}\text { Respondents } \\
\text { education }\end{array}$} & Illiterate & 59.2 & 40.8 & \multirow{4}{*}{321.37} & \multirow{4}{*}{.000} & 5.9 & 94.1 & \multirow{4}{*}{114.4} & \multirow{4}{*}{.000} & 8.5 & 91.5 & \multirow{4}{*}{10.94} & \multirow{4}{*}{.012} \\
\hline & Primary & 36.1 & 63.9 & & & 3.1 & 96.9 & & & 8.0 & 92.0 & & \\
\hline & Secondary & 12.9 & 87.1 & & & 1.8 & 98.2 & & & 7.3 & 92.7 & & \\
\hline & Higher & .7 & 99.3 & & & .5 & 99.5 & & & 5.7 & 94.3 & & \\
\hline
\end{tabular}

Table 2 Contd. 


\begin{tabular}{|c|c|c|c|c|c|c|c|c|c|c|c|c|c|}
\hline \multirow{2}{*}{\multicolumn{2}{|c|}{$\begin{array}{c}\text { Background } \\
\text { characteristics }\end{array}$}} & \multicolumn{4}{|c|}{$\begin{array}{c}\text { Ever heard } \\
\text { of HIV/AIDS }\end{array}$} & \multicolumn{4}{|c|}{$\begin{array}{c}\text { HIV/AIDS transmission } \\
\text { knowledge }\end{array}$} & \multicolumn{4}{|c|}{$\begin{array}{c}\text { HIV/AIDS prevention } \\
\text { knowledge }\end{array}$} \\
\hline & & $\begin{array}{l}\text { No } \\
(\%)\end{array}$ & $\begin{array}{l}\text { Yes } \\
(\%)\end{array}$ & $\chi^{2}$ & P-value & $\begin{array}{l}\text { No } \\
(\%)\end{array}$ & $\begin{array}{l}\text { Yes } \\
(\%)\end{array}$ & $\chi^{2}$ & P-value & $\begin{array}{l}\text { No } \\
(\%)\end{array}$ & $\begin{array}{l}\text { Yes } \\
(\%)\end{array}$ & $\chi^{2}$ & P-value \\
\hline \multirow{4}{*}{$\begin{array}{l}\text { Husbands } \\
\text { Education }\end{array}$} & Illiterate & 51.8 & 48.2 & \multirow{4}{*}{2224.78} & \multirow{4}{*}{.000} & 4.3 & 95.7 & \multirow{4}{*}{48.72} & \multirow{4}{*}{.000} & 8.1 & 91.9 & \multirow{4}{*}{7.05} & \multirow{4}{*}{.070} \\
\hline & Primary & 34.3 & 65.7 & & & 2.9 & 97.1 & & & 7.2 & 92.8 & & \\
\hline & Secondary & 16.4 & 83.6 & & & 2.0 & 98.0 & & & 7.9 & 92.1 & & \\
\hline & Higher & 5.0 & 95.0 & & & 1.3 & 98.7 & & & 6.3 & 93.7 & & \\
\hline \multirow{2}{*}{ Religion } & Islam & 29.6 & 70.4 & \multirow{2}{*}{14.27} & \multirow{2}{*}{.000} & 2.6 & 97.4 & \multirow{2}{*}{1.09} & \multirow{2}{*}{.173} & 7.2 & 92.8 & \multirow{2}{*}{7.14} & \multirow{2}{*}{.005} \\
\hline & Others & 34.1 & 65.9 & & & 2.1 & 97.9 & & & 9.5 & 90.5 & & \\
\hline \multirow{4}{*}{$\begin{array}{l}\text { Husbands } \\
\text { Occupation }\end{array}$} & Agriculture & 48.0 & 52.0 & \multirow{4}{*}{1359.23} & \multirow{4}{*}{.000} & 3.5 & 96.5 & \multirow{4}{*}{19.50} & \multirow{4}{*}{.000} & 8.1 & 91.9 & \multirow{4}{*}{7.11} & \multirow{4}{*}{.068} \\
\hline & $\begin{array}{l}\text { Service } \\
\text { Holder }\end{array}$ & 14.9 & 85.1 & & & 1.8 & 98.2 & & & 6.6 & 93.4 & & \\
\hline & Business & 22.3 & 77.7 & & & 2.5 & 97.5 & & & 7.7 & 92.3 & & \\
\hline & Others & 35.4 & 64.6 & & & 3.1 & 96.9 & & & 8.0 & 92.0 & & \\
\hline \multirow{2}{*}{$\begin{array}{c}\text { Current marital } \\
\text { Status }\end{array}$} & Married & 29.2 & 70.8 & \multirow{2}{*}{79.59} & \multirow{2}{*}{.000} & 2.5 & 97.5 & \multirow{2}{*}{7.14} & \multirow{2}{*}{.009} & 7.4 & 92.6 & \multirow{2}{*}{.89} & \multirow{2}{*}{.194} \\
\hline & Others & 42.8 & 57.2 & & & 4.3 & 95.7 & & & 8.5 & 91.5 & & \\
\hline Access to mass & No & 53.2 & 46.8 & 253445 & 000 & 4.3 & 95.7 & 1570 & 000 & 8.3 & 91.7 & 4 & 023 \\
\hline Media & Yes & 15.9 & 84.1 & 2534.43 & .000 & 2.0 & 98.0 & 45.19 & (.000 & 7.2 & 92.8 & 4.11 & . 025 \\
\hline & Poor & 52.8 & 47.2 & & & 4.6 & 95.4 & & & 8.8 & 91.2 & & \\
\hline Status & Middle & 16.2 & 83.8 & 1883.31 & .000 & 1.9 & 98.1 & 46.98 & .000 & 7.1 & 92.9 & 6.23 & .044 \\
\hline & Rich & 39.9 & & & & 3.3 & 96.7 & & & 7.6 & 92.4 & & \\
\hline Current use of & No & 33.8 & 66.2 & 74602 & 000 & 2.8 & 97.2 & 161 & 114 & 7.5 & 92.5 & 006 & 413 \\
\hline contraceptives & Yes & 27.5 & 72.5 & 14.002 & .000 & 2.4 & 97.6 & 1.01 & .114 & 7.4 & 92.6 & 0.00 & . .413 \\
\hline
\end{tabular}

Findings in the Table 2 reveal that the variables-respondents current age, type of place of residence, division, husbands' education, religion, husbands occupation, and current marital status, access to mass media, socioeconomic status and current contraceptive use are significantly associated with HIV/AIDS awareness at 5\% level of significance. In addition, the variables type of place of residence, division, respondents' education, husband's education and occupation, current marital status, access to mass media and socio-economic status are found to have a significant association with the comprehensive knowledge of HIV/AIDS transmission. Moreover, the explanatory variables which are found to have a significant association with HIV/AIDS prevention knowledge are type of place of residence, division, respondents' education, religion, access to mass media and socio-economic status. 
In the Table 2, the differentials by background characteristics of the awareness of HIV/AIDS indicate that $99.3 \%$ of the respondents who are highly educated are aware of AIDS followed by those whose husbands are highly educated (95.0\%). Only 46.8\% respondents who have no access to mass media are aware of AIDS, followed by those who are poor (47.2\%). Therefore, mass media campaigns should be strengthened to increase the current level of awareness of HIV/AIDS. Differentials by background characteristics of the HIV/AIDS transmission and prevention knowledge among the respondents who have heard of AIDS reveals that the level of HIV/AIDS transmission knowledge is high among highly educated respondents (99.5\%) followed by those whose husbands are highly educated (98.7\%) and HIV transmission knowledge is found relatively lower among the respondents whose husbands are illiterate (95.7\%). Moreover, HIV/AIDS prevention knowledge is found high among the respondents of Dhaka division (95.4\%) followed by the respondents living in Rajshahi region (94.9\%) and prevention knowledge is found low among the respondents living in Sylhet division $(90.1 \%)$ and among non-Muslim $(90.5 \%)$. The bivariate analysis reflects that the explanatory variables respondents current age, place of residence, division, respondents education, husbands education, religion, husbands occupation, current marital status, access to mass media, socio-economic status and use of contraceptives are found to have significant association with the awareness of HIV/AIDS. In addition, type of place of residence, division, respondents' education, husbands' education and occupation, current marital status, access to mass media and socio-economic status are found significant association with HIV/AIDS transmission knowledge. The results further illustrate that type of place of residence, division, respondents education, religion, access to mass media and socio-economic status are found to have significant association with the prevention knowledge of HIV/AIDS in Bangladesh.

Besides association of attributes, application of multivariate analysis is inevitable to identify the risk or prognostic factors of HIV/AIDS awareness, its transmission and prevention knowledge. To carry out the multivariate analysis, the hierarchical logistic regression is appropriate because this method does not require any distributional assumptions of the explanatory variables. Moreover, it can be used for qualitative, 
quantitative and mix of both qualitative and quantitative variables. The results of hierarchical logistic regression analysis about awareness of HIV/AIDS for all models are presented in the Table 3. The findings of hierarchical logistic regression in the Table 3 elucidate that the age of women has positive significant impact on the awareness of HIV/AIDS. The respondents of the age group 25-29 are more knowledgeable about HIV/AIDS. The odds ratio 1.95 indicates that the respondents of the age group 25-29 are approximately 2 times on an average more exposed to have knowledge about HIV/AIDS compared to the reference age group of 15-19 years. Result also reveals that the respondents who are highly educated are about 47 times more knowledgeable than those who are illiterate and the findings are in the same line for all the models. Husbands' educational level is also found to have significant impact on HIV/AIDS awareness. The odds ratio 2.2 states that the respondents whose husbands are highly educated are 2.2 times more likely to know about HIV/AIDS than those whose husbands are illiterate. In an earlier study it was found that knowledge of AIDS was strongly and positively associated with the education of female adolescents and their husbands and varied significantly across different parts of the country [11]. Like education, husband's occupation is also found to have significant impact on the awareness of HIV/AIDS.

Table 3. Hierarchical Logistic Regression estimates of the awareness of HIV/AIDS among ever-married women in Bangladesh

\begin{tabular}{|c|c|c|c|c|c|c|c|}
\hline \multirow{2}{*}{\multicolumn{2}{|c|}{$\begin{array}{l}\text { Background } \\
\text { Characteristics }\end{array}$}} & \multirow{2}{*}{$\begin{array}{c}\text { Model } 1 \\
\text { Odds } \\
\text { Ratio }\end{array}$} & \multirow{2}{*}{$\begin{array}{c}\text { Model } 2 \\
\text { Odds } \\
\text { Ratio }\end{array}$} & \multirow{2}{*}{$\begin{array}{c}\text { Model } 3 \\
\text { Odds } \\
\text { Ratio }\end{array}$} & \multicolumn{2}{|c|}{ Model 4} & \multirow{2}{*}{$\begin{array}{c}\begin{array}{c}\text { Confidence } \\
\text { Interval }\end{array} \\
\text { Upper }\end{array}$} \\
\hline & & & & & $\begin{array}{l}\text { Odds } \\
\text { Ratio }\end{array}$ & Lower & \\
\hline \multirow{4}{*}{$\begin{array}{l}\text { Respondents } \\
\text { current age }\end{array}$} & 15-19 & - & - & - & - & - & - \\
\hline & $20-24$ & $1.391 * * *$ & $1.598 * * *$ & $1.612 * * *$ & $1.575^{* * *}$ & 1.337 & 1.857 \\
\hline & $25-29$ & $1.357 * * *$ & $1.996 * * *$ & $2.017 * * *$ & $1.950 * * *$ & 1.655 & 2.297 \\
\hline & $30+$ & $.729 * * *$ & $1.614 * * *$ & $1.596^{* * *}$ & $1.567 * * *$ & 1.352 & 1.815 \\
\hline \multirow{4}{*}{$\begin{array}{l}\text { Respondents } \\
\text { education }\end{array}$} & Illiterate & \multirow{4}{*}{--} & - & - & - & - & - \\
\hline & Primary & & $2.112 * * *$ & $2.070 * * *$ & $2.037 * * *$ & 1.842 & 2.252 \\
\hline & Secondary & & $5.417 * * *$ & $5.541 * * *$ & $5.419 * * *$ & 4.766 & 6.161 \\
\hline & Higher & & $49.407 * * *$ & $48.685^{* * *}$ & $47.299 * * *$ & 25.537 & 87.606 \\
\hline \multirow{4}{*}{$\begin{array}{l}\text { Husbands } \\
\text { education }\end{array}$} & Illiterate & \multirow[t]{4}{*}{--} & - & - & - & - & - \\
\hline & Primary & & $1.137 *$ & $1.145^{* *}$ & $1.137 *$ & 1.028 & 1.257 \\
\hline & Secondary & & $1.679 * * *$ & $1.683^{* * *}$ & $1.700 * * *$ & 1.510 & 1.914 \\
\hline & Higher & & $2.102 * * *$ & $2.156^{* * * *}$ & $2.200 * * *$ & 1.749 & 2.766 \\
\hline
\end{tabular}




\begin{tabular}{|c|c|c|c|c|c|c|c|}
\hline \multirow{2}{*}{\multicolumn{2}{|c|}{$\begin{array}{c}\text { Background } \\
\text { Characteristics }\end{array}$}} & \multirow{2}{*}{$\begin{array}{c}\text { Model } 1 \\
\text { Odds } \\
\text { Ratio }\end{array}$} & \multirow{2}{*}{$\begin{array}{c}\text { Model } 2 \\
\text { Odds } \\
\text { Ratio }\end{array}$} & \multirow{2}{*}{$\begin{array}{c}\text { Model } 3 \\
\text { Odds } \\
\text { Ratio }\end{array}$} & \multicolumn{2}{|c|}{ Model 4} & \multirow{2}{*}{$\begin{array}{c}\begin{array}{c}\text { Confidence } \\
\text { Interval }\end{array} \\
\text { Upper }\end{array}$} \\
\hline & & & & & Odds & J & \\
\hline \multirow{4}{*}{$\begin{array}{l}\text { Husbands } \\
\text { occupation }\end{array}$} & Agriculture & -- & - & - & - & - & - \\
\hline & Service & & $1.973 * * *$ & $1.796 * * *$ & $1.804 * * *$ & 1.597 & 2.036 \\
\hline & Business & & $1.632 * * *$ & $1.514 * * *$ & $1.504 * * *$ & 1.338 & 1.690 \\
\hline & Others & & $1.577 * * *$ & $1.463 * * *$ & $1.469 * * *$ & 1.317 & 1.638 \\
\hline Access to mass media & Yes & -- & $2.673 * * *$ & 2.658 *** & $2.655 * * *$ & 2.405 & 2.930 \\
\hline \multirow{3}{*}{ Socio-economic status } & Poor & -- & - & - & - & - & - \\
\hline & Middle & & $1.505 * * *$ & $1.390 * * *$ & $1.388 * * *$ & 1.229 & 1.567 \\
\hline & Rich & & 1.075 & 1.055 & 1.049 & .942 & 1.169 \\
\hline $\begin{array}{l}\text { Type of place of } \\
\text { residence }\end{array}$ & Rural & & & $.710^{* * * *}$ & $.709^{* * *}$ & .642 & .784 \\
\hline \multirow{7}{*}{ Division } & Rangpur & -- & -- & - & - & - & - \\
\hline & Barisal & & & $1.687 * * *$ & $1.716^{* * *}$ & 1.473 & $1.716^{* * * *}$ \\
\hline & Chittagong & & & 0.957 & 0.991 & 0.859 & 0.991 \\
\hline & Dhaka & & & $1.587 * * *$ & $1.613 * * *$ & 1.394 & $1.613 * * *$ \\
\hline & Khulna & & & $2.146 * * *$ & $2.174 * * *$ & 1.873 & $2.174 * * *$ \\
\hline & Rajshahi & & & 0.974 & 0.972 & 0.843 & 0.972 \\
\hline & Sylhet & & & 1.156 & $1.217 *$ & 1.049 & $1.217 *$ \\
\hline Religion & Non-Muslim & -- & & $.748^{* * * *}$ & $.740^{* * *}$ & .645 & .849 \\
\hline $\begin{array}{c}\text { Current contraceptive } \\
\text { use }\end{array}$ & Yes & -- & & & $1.242 * * *$ & .766 & 1.073 \\
\hline Current marital status & Separated & -- & & & .907 & 1.138 & 1.356 \\
\hline \multicolumn{2}{|c|}{-2 Log Likelihood } & 19559.663 & 14728.806 & 14448.781 & \multicolumn{3}{|c|}{14417.203} \\
\hline \multicolumn{2}{|c|}{ Chi-square } & $281.554 * * *$ & $5112.412 * * *$ & $5392.437 * * *$ & \multicolumn{3}{|c|}{$5424.015^{* * *}$} \\
\hline \multicolumn{2}{|c|}{ Error Reduction } & 281.554 & 4830.858 & 280.025 & \multicolumn{3}{|c|}{31.578} \\
\hline
\end{tabular}

The hierarchical logistic regression shows that the respondents whose husbands are service holder are approximately 1.81 times more knowledgeable about HIV/AIDS relative to their agriculturist counterparts. The respondents who have access to mass media are 2.66 times more knowledgeable about HIV/AIDS compared to the respondents who do not have any access to media. In a similar study it is found that knowledge on HIV/AIDS was higher among relatively older and urban residents who had access to television or radio and whose husbands were using condom [11]. The result shows that the respondents belong to middle-class stratum have more knowledge than other socio-economic groups. As expected, place of residence has a 
significant influence on the awareness of HIV/AIDS in Bangladesh. The respondents who live in the rural areas are $29 \%$ less likely to know about HIV/AIDS. The output of multivariate analysis also reveals that Chittagong division does not have any significant effect on the awareness of HIV/AIDS. However, overall result shows that administrative division has significant influence on the awareness of HIV/AIDS. The odds ratio 2.17 indicates that the respondents of Khulna division are 2.17 times more likely to know about HIV/AIDS as compared to the respondents of Rangpur region. The Muslim respondents are more knowledgeable than their non-Muslim counterparts. The respondents who use contraceptives are 1.24 times more likely to know about the HIV/AIDS than the respondents who does not use contraceptive methods and the coefficient is found significant. Based on the values of $\chi^{2}$ test statistic and error reduction, it can be concluded that model 4 in the Table 3 is the best for awareness of HIV/AIDS among ever married women in Bangladesh.

For assessing the impact of explanatory variables on the HIV/AIDS transmission knowledge, the results of hierarchical logistic regression are presented in the Table 4. Findings of hierarchical logistic regression reveal that education of the respondents has an immense influence on HIV transmission knowledge like awareness. The result also shows that the respondents who are highly educated are 10.39 times more likely to know about HIV transmission knowledge compared to those who are illiterate. 
Table 4. Hierarchical Logistic Regression estimates of HIV/AIDS transmission knowledge among ever married women who have heard of AIDS in Bangladesh

\begin{tabular}{|c|c|c|c|c|c|c|c|}
\hline \multirow{2}{*}{\multicolumn{2}{|c|}{$\begin{array}{c}\text { Background } \\
\text { Characteristics }\end{array}$}} & \multirow{2}{*}{$\begin{array}{c}\text { Model } 1 \\
\text { Odds } \\
\text { Ratio }\end{array}$} & \multirow{2}{*}{$\begin{array}{l}\text { Model } 2 \\
\text { Odds } \\
\text { Ratio }\end{array}$} & \multirow{2}{*}{$\begin{array}{c}\text { Model } 3 \\
\text { Odds } \\
\text { Ratio }\end{array}$} & \multirow{2}{*}{$\begin{array}{c}\text { Model } 4 \\
\text { Odds } \\
\text { Ratio }\end{array}$} & \multicolumn{2}{|c|}{$\begin{array}{c}\text { Confidence } \\
\text { Interval }\end{array}$} \\
\hline & & & & & & \multirow{2}{*}{$\begin{array}{c}\text { Lower } \\
-\end{array}$} & \multirow{2}{*}{$\begin{array}{c}\text { Upper } \\
-\end{array}$} \\
\hline Socio- & Poor & - & - & - & - & & \\
\hline economic & Middle & $2.521 * * *$ & $1.512 * *$ & $1.396^{*}$ & $1.392 *$ & .965 & 2.006 \\
\hline status & Rich & $1.425^{* *}$ & 1.195 & 1.154 & 1.158 & .826 & 1.623 \\
\hline \multirow{4}{*}{$\begin{array}{l}\text { Respondents } \\
\text { education }\end{array}$} & Illiterate & \multirow{4}{*}{--} & - & - & - & - & - \\
\hline & Primary & & $1.829 * * *$ & $1.818 * * *$ & $1.795 * * *$ & 1.324 & 2.435 \\
\hline & Secondary & & $2.951 * * *$ & $2.912 * * *$ & $2.849 * * *$ & 2.016 & 4.026 \\
\hline & Higher & & $11.015 * * *$ & $10.712 * * *$ & $10.389 * * *$ & 4.421 & 24.417 \\
\hline \multirow{4}{*}{$\begin{array}{l}\text { Husband } \\
\text { education }\end{array}$} & Illiterate & -- & - & - & - & - & - \\
\hline & Primary & & 1.064 & 1.081 & 1.073 & .785 & 1.468 \\
\hline & Secondary & & 1.117 & 1.072 & 1.072 & .755 & 1.522 \\
\hline & Higher & & .848 & .805 & .811 & .485 & 1.354 \\
\hline \multirow{4}{*}{$\begin{array}{l}\text { Husband } \\
\text { occupation }\end{array}$} & Agriculture & -- & - & - & - & - & - \\
\hline & Service holder & & 1.155 & 1.222 & 1.223 & .856 & 1.748 \\
\hline & Business & & .954 & 1.000 & 1.004 & .710 & 1.421 \\
\hline & Others & & 1.079 & 1.102 & 1.102 & .783 & 1.550 \\
\hline $\begin{array}{c}\text { Access to } \\
\text { mass media }\end{array}$ & Yes & -- & $1.336^{*}$ & $1.326^{*}$ & $1.330^{*}$ & .982 & 1.800 \\
\hline $\begin{array}{l}\text { Type of place } \\
\text { of resident }\end{array}$ & Rural & -- & & .901 & .893 & .677 & 1.178 \\
\hline \multirow{7}{*}{ Division } & Rangpur & -- & -- & - & - & - & - \\
\hline & Barisal & & & 1.082 & 1.087 & 0.673 & 1.757 \\
\hline & Chittagong & & & 0.841 & 0.842 & 0.541 & 1.312 \\
\hline & Dhaka & & & 0.945 & 0.948 & 0.615 & 1.46 \\
\hline & Khulna & & & 1.552 & 1.565 & 0.968 & 2.53 \\
\hline & Rajshahi & & & 1.299 & 1.299 & 0.793 & 2.127 \\
\hline & Sylhet & & & $0.466 * * *$ & $0.472 * * *$ & 0.310 & 0.713 \\
\hline $\begin{array}{c}\text { Current } \\
\text { marital status }\end{array}$ & Separated & -- & -- & -- & .778 & .501 & 1.207 \\
\hline \multicolumn{2}{|c|}{-2 Log Likelihood $^{\epsilon}$} & 2662.978 & 2572.119 & 2537.087 & \multicolumn{2}{|c|}{2535.901} & \\
\hline \multicolumn{2}{|c|}{ Chi-square } & $42.805^{* * *}$ & $133.664 * * *$ & $168.696^{* * *}$ & \multicolumn{2}{|c|}{$169.881 * * *$} & \\
\hline \multicolumn{2}{|c|}{ Error Reduction } & 42.805 & 90.859 & 35.032 & 1.186 & & \\
\hline \multicolumn{8}{|c|}{ Note: Reference category $(-) ; * * *<0.001 ; * * p<0.01 ; * p<0.05$} \\
\hline
\end{tabular}


Findings of this study further illustrate that among the administrative divisions, the Sylhet division shows only significant effect on HIV transmission knowledge. But the respondents of Khulna division have more knowledge about HIV than the respondents of other divisions. Thus, it can be said that the administrative division has indirect influence on the awareness to HIV transmission. Access to mass media has a marginal impact on the knowledge of HIV transmission. The respondents with middle socio-economic status are 1.39 times more knowledgeable about HIV transmission than the respondents who are poor. The hierarchical logistic regression reveals that the other predictor variables such as husband's educational level and occupation have indirect effect on HIV transmission knowledge. The values of test statistic $\chi^{2}$ along with error reduction in the Table 4 indicate that model 4 is the best for HIV/AIDS transmission knowledge among the respondents in Bangladesh. In addition to HIV/AIDS transmission, the results of multivariate hierarchical logistic regression about HIV/AIDS prevention knowledge are also shown in the Table 5 for comparative study.

Table 5. Hierarchical Logistic Regression estimates of HIV/AIDS prevention knowledge among ever-married women who have heard of AIDS in Bangladesh

\begin{tabular}{ccccccc}
\hline & & Model 1 & Model 2 & \multicolumn{2}{c}{ Model 3 } & \multicolumn{2}{c}{ Confidence Interval } \\
\hline $\begin{array}{c}\text { Background } \\
\text { characteristics }\end{array}$ & & Odds & Odds & Odds & Lower & Upper \\
& Ratio & Ratio & Ratio & & \\
\hline \multirow{3}{*}{ Socio-economic status } & Middle & $1.269^{* *}$ & 1.155 & 1.098 & .871 & 1.386 \\
& Rich & 1.172 & 1.140 & 1.127 & .891 & 1.425 \\
\hline \multirow{5}{*}{ Respondents education } & Illiterate & & - & - & - & - \\
& Primary & & $\mathbf{1 . 0 6 2}$ & 1.058 & .849 & 1.320 \\
& Secondary & & 1.140 & 1.175 & .953 & 1.450 \\
& Higher & & $1.472^{* * *}$ & $1.434 * *$ & 1.069 & 1.923 \\
\hline
\end{tabular}

Table 5 Contd. 


\begin{tabular}{|c|c|c|c|c|c|c|}
\hline & & Model 1 & Model 2 & Model 3 & Confic & e Interval \\
\hline \multicolumn{2}{|l|}{$\begin{array}{l}\text { Background } \\
\text { characteristics }\end{array}$} & \multirow[t]{2}{*}{$\begin{array}{l}\text { Odds } \\
\text { Ratio }\end{array}$} & $\begin{array}{l}\text { Odds } \\
\text { Ratio }\end{array}$ & $\begin{array}{l}\text { Odds } \\
\text { Ratio }\end{array}$ & Lower & Upper \\
\hline Access to mass media & Yes & & 1.049 & .993 & .817 & 1.207 \\
\hline \multirow[t]{4}{*}{ Place of residence } & Rural & & & $.860^{*}$ & .735 & 1.006 \\
\hline & Rangpur & & & - & - & - \\
\hline & Barisal & & & $0.627 * * *$ & 0.524 & 0.75 \\
\hline & Chittagong & & & 1.13 & 0.937 & 1.363 \\
\hline \multirow[t]{4}{*}{ Division } & Dhaka & & & 1.014 & 0.842 & 1.222 \\
\hline & Khulna & & & 1.12 & 0.918 & 1.367 \\
\hline & Rajshahi & & & 1.139 & 0.931 & 1.394 \\
\hline & Sylhet & & & $0.619 * * * *$ & 0.51 & 0.751 \\
\hline \multicolumn{2}{|l|}{-2 Log likelihood } & 5979.083 & 5970.482 & 5897.972 & & \\
\hline \multicolumn{2}{|l|}{ Chi-square } & $6.015 * *$ & $14.616 * *$ & $87.127 * * *$ & & \\
\hline \multicolumn{2}{|l|}{ Error Reduction } & 6.015 & 8.602 & 72.510 & & \\
\hline
\end{tabular}

Findings in the Table 5 show that the administrative division has highly significant influence on the awareness of HIV/AIDS prevention. The respondents of Dhaka and Rajshahi divisions are found to have more knowledge about HIV/AIDS prevention than other divisions. The respondents' education has a significant impact on the HIV/AIDS prevention knowledge. The odds ratio of the hierarchical logistic regression reveals that the respondents who are highly educated are 1.43 times more likely to know about HIV/AIDS prevention knowledge. The type of place of residence is found to have significant impact on HIV/AIDS prevention knowledge. The odds ratio implies that urban respondents are more knowledgeable about HIV/AIDS prevention than the rural respondents. The finding also reveals that the predictor variables access to mass media and socio-economic status have indirect 
impact on the HIV/AIDS prevention knowledge. The values of Chi-square test statistic and error reduction in the Table 5 shows that model 3 is the best for HIV/AIDS prevention knowledge among ever married women in Bangladesh.

\section{Conclusion}

Among the factors considered in this analysis, the respondents' education is found very significant predictor of HIV/AIDS. A major factor that has a significant influence on HIV/AIDS awareness and its transmission and prevention knowledge is respondents' education. Earlier studies conducted in Bangladesh confirmed that knowledge on AIDS was strongly and positively associated with education [12-13]. This result is in the same direction with another study [14]. In Bangladesh, the people do not like to talk about HIV/AIDS as it is related to sexuality, so there is a very narrow space to know about HIV/AIDS. Hence, the people who are secondary or highly educated can able to read more extra academic papers, pamphlets and story books from which they can come to know about HIV/AIDS epidemic and also can gather knowledge about its transmission and prevention process easily. Like education, access to mass media is also an important factor that has a significant influence on the awareness of HIV/AIDS because education and mass media contact are interrelated. A recent study also suggested that mass media campaign may be an important tool to increase the knowledge of HIV/AIDS [15]. Media like TVs, Newspaper, and Radio can play a vital role to increase the awareness level of HIV/AIDS. Nowadays, most of the people in Bangladesh and around the world have a tendency on media as an entertainment. As most of the media proclaim different awareness raising program including HIV/AIDS, the mass people can come to know about such kind of disease from mass media contact. Hence, it can be concluded that

access to mass media is the key factor that have a great influence on awareness of HIV/AIDS.

Type of place of residence has significant and positive influence on the awareness of HIV/AIDS and it has also an impact on its transmission and prevention as well. As urban areas are adorned with modern facilities with hospitals, schools, universities, 
community centers and also greater mass media exposure, hence the respondents who live in the urban area are usually more knowledgeable about HIV/AIDS. Therefore, campaign should be strengthened among the couples in the vast of rural areas about the awareness of HIV/AIDS.

Husbands' occupation has an influence on the primary awareness of HIV/AIDS. Administrative division is found to have a significant influence on the awareness of HIV/AIDS and it is also significant for both HIV/AIDS transmission and prevention knowledge. This may be due to the wide variations of socio-economic and geographical settings among different regions of the country. Findings show that the respondents of Khulna division are more knowledgeable than other divisions but in case of HIV prevention knowledge, the Dhaka division is found to have relatively better since most-at-risk groups of HIV/AIDS are living in Dhaka city[16], the overall awareness level should be increased in Dhaka mega city. Comparative findings confirm that the respondents of Khulna city are more knowledgeable about HIV/AIDS. Like respondents education, partner's education is found positively associated with the awareness of HIV/AIDS. Result reflects that the respondents whose husbands are highly educated are more knowledgeable about HIV/AIDS. This may be due to fact that the respondents may discuss the matter regarding sexuality and as well as the STI's which is related to HIV/AIDS. Finding thus suggests that the respondents whose husbands are highly educated can provide more information and hence become more knowledgeable about the consequences of HIV/AIDS.

The age of the respondents has an impact on the awareness of HIV/AIDS but does not have influence on the comprehensive knowledge on HIV/AIDS. Socio-economic status of the respondents is also has the significant effect on the awareness of HIV/AIDS. Couples who use contraceptive methods are more knowledgeable about HIV/AIDS [17], which indicates that the use of contraceptives has a significant influence on the awareness level of HIV/AIDS but it does not have an influence on HIV/AIDS transmission and prevention knowledge. Marital status of the respondents has a significant influence on the variations of HIV/AIDS awareness. Thus, findings at a glance confirmed that the education, access to mass media, age, type of place of 
residence, husband's education and occupation, socio economic status and contraceptive use have significant influence on the awareness of HIV/AIDS in Bangladesh.

Therefore, based on the findings obtained from the multidimensional analyses it can be suggested that fruitful steps should be launched by concerned authorities to increase the educational level and socio economic conditions of the people across the country. Moreover, electronic and print media campaign should be strengthened regarding the consequences of HIV/AIDS. In addition, regional disparities in the context of education, socio-economic condition and other facilities should be reduced in a great extent. Furthermore, increase of contraceptive prevalence rate (CPR) could be an important significant factor for raising the awareness level of HIV/AIDS and reduction of its spread.

Finally, it can be concluded that proper implementation of the recommendations given above likely to raise the awareness level, transmission and prevention knowledge of HIV/AIDS, which could also be a best course of action for the reduction of its spread in the densely populated country of Bangladesh.

\section{References}

[1] J. Shaman and M. Kohn, Proc. Natl. Acad. Sci. U. S. A.,2009, 106(9), 3243.

[2] R. Granich, B. Williams, J. Montaner, and J. M. Zuniga, The Lancet, 2017, 390(10092), 341.

[3] https://www.unaids.org/en/resources/documents/2016/prevention-gap (Last accessed on January, 2021).

[4] J. D. Smith and K. L. Tan, Otolaryngol. Clin. North Am., 2018, 51( 3), 659.

[5] ttps://www.unaids.org/en/dataanalysis/knowyourresponse/HIVdata_estimates (Last accessed January, 2021).

[6] O. Faruk, N. Begum, and S. Rana, Int. J. Data Sci. Technol., 2017, 3(6), 56.

[7] Mitra and Associates and Macro International, Bangladesh Demographic and Health Survey, Dhaka, Bangladesh and Calverton, Maryland, USA, 2007. 
[8] Mitra and Associates and Macro International, Bangladesh Demographic and Health Survey, Dhaka, Bangladesh and Calverton, Maryland, USA, 2011.

[9] Mitra and Associates and Macro International, Bangladesh Demographic and Health Survey, Dhaka, Bangladesh and Calverton, Maryland, USA, 2014.

[10] P. Estébanez, N. K. Russell, M. D. Aguilar, I. Cifuentes, M. V. Zunzunegui, and K. Mcpherson, Eur. J. Epidemiol., 2001, 17(6), 573.

[11] M. M. Rahman, M. Kabir, and M. Shahidullah, http://www.ayubmed.edu.pk/JAMC/PAST/21-3/Mizan.pdf. (Last accessed: January, 2021).

[12] R. Mm, M. Kabir, and M. Shahidullah, "Knowledge of AIDS and condom as a preventive measure against AIDS among married males and factors influencing it in Bangladesh."

[13] N. Islam Mondal, R. Islam, O. Rahman, S. Rahman, and N. Hoque, World J. AIDS, 2012, 2(4), 312.

[14] J. Health Popul Nutr; Khan and M. J. H. P. Nutr, Evidence from the Bangladesh Demographic and Health Survey Data, 2002, $20(2), 130$.

[15] M. A. Khan, M. Rahman, P. A. Khanam, Barkat-e-Khuda, T. T. Kane, and A. Ashraf, Int. J. STD AIDS, 1997, 8(11): 688.

[16] M. Nazrul Islam Mondal, M. Mahfuzar Rahman, M. Obaidur Rahman, and M. Naznin Akter, Food Public Heal., 2012, 2(3), 73.

[17] S. Mou, F. Bhuiya, and S. S. Islam, Int. J. Adv. Med. Heal. Res., 2015, 2(1), 9.

The Chittagong Univ. J. Sc. Vol. 43(1), 2021 